\title{
Neural Correlates of an Injury-Free Model of Central Sensitization Induced by Opioid Withdrawal in Humans
}

\author{
Vishvarani Wanigasekera, ${ }^{1}$ Michael C. H. Lee, ${ }^{1,2}$ Richard Rogers, ${ }^{1}$ Philip Hu, ${ }^{1}$ and Irene Tracey ${ }^{1}$ \\ ${ }^{1}$ Centre for Functional Magnetic Resonance Imaging of the Brain (FMRIB), Nuffield Department of Clinical Neurosciences, Division of Anaesthetics, \\ University of Oxford, John Radcliffe Hospital, Headington, Oxford OX3 9DU, United Kingdom, and 2Department of Medicine, Division of Anaesthesia, \\ University of Cambridge, Cambridge CB2 2QQ, United Kingdom
}

Preclinical evidence suggests that opioid withdrawal induces central sensitization (CS) that is maintained by supraspinal contributions from the descending pain modulatory system (DPMS). Here, in healthy human subjects we use functional magnetic resonance imaging to study the supraspinal activity during the withdrawal period of the opioid remifentanil. We used a crossover design and thermal stimuli on uninjured skin to demonstrate opioid withdrawal-induced hyperalgesia $(\mathrm{OIH})$ without a CS-inducing peripheral stimulus. Saline was used in the control arm to account for effects of time. OIH in this injury-free model was observed in a subset of the healthy subjects (responders). Only in these subjects did opioid infusion and withdrawal induce a rise in activity in the mesencephalic-pontine reticular formation (MPRF), an area of the DPMS that has been previously shown to be involved in states of CS in humans, which became significant during the withdrawal phase compared with nonresponders. Paradoxically, this opioid withdrawal-induced rise in MPRF activity shows a significant negative correlation with the behavioral OIH score indicating a predominant inhibitory role of the MPRF in the responders. These data illustrate that in susceptible individuals central mechanisms appear to regulate the expression of OIH in humans in the absence of tissue injury, which might have relevance for functional pain syndromes where a peripheral origin for the pain is difficult to identify.

\section{Introduction}

Increased pain perception to a noxious stimulus (hyperalgesia) is a key manifestation of central sensitization (CS) states most clearly characterized by increased excitability of neurons in the spinal dorsal horn. This excitability is regulated by peripheral nociceptive input from injured tissue (Ji et al., 2003) as well as by the dynamic shift between the descending inhibitory and facilitatory inputs from the brainstem (Heinricher et al., 2009). Functional pain syndromes (FPS), such as fibromyalgia and irritable bowel syndrome, are a group of chronic pain disorders characterized by increased pain sensitivity in the absence of demonstrable tissue injury. It has been proposed that despite lack of an initiating peripheral input, CS and abnormalities in the descending pain modulation are responsible for this heightened pain sensitivity (Julien et al., 2005; Tillisch and Mayer, 2005; Yunus, 2007; Berman et al., 2008).

Data from animal studies support the role of descending pain facilitation from brainstem nuclei in the development and maintenance of CS. This is observed in models of chronic pain states

\footnotetext{
Received Oct. 15, 2010; revised Dec. 13, 2010; accepted Dec. 16, 2010.

This work was supported by grants from Medical Research Council of Great Britain and Northern Ireland (FMRIB (entre and M.C.H.L.) and Wellcome Trust (V.W. and I.T.)

This article is freely available online through the $J$ Neurosci Open Choice option.

Correspondence should be addressed to Vishvarani Wanigasekera, Centre for Functional Magnetic Resonance Imaging of the Brain (FMRIB), Nuffield Department of Clinical Neurosciences, Division of Anaesthetics, University of Oxford, John Radcliffe Hospital, Headington, Oxford OX3 9DU, United Kingdom. E-mail: vishwani@fmrib.ox.ac.uk.

DOI:10.1523/JNEUROSCI.5412-10.2011

Copyright (C) 2011 the authors $\quad 0270-6474 / 11 / 312835-08 \$ 15.00 / 0$
}

(Gebhart, 2004) as well as in models of injury-free hyperalgesia, where hyperalgesia is induced by opioid withdrawal (Kaplan and Fields, 1991).

Emerging evidence from human imaging studies indicate that the brainstem mesencephalic-pontine reticular formation (MPRF) activity is a neural correlate of states of CS induced by capsaicin (Iannetti et al., 2005; Zambreanu et al., 2005; Lee et al., 2008).

Therefore, we hypothesized that in humans, brainstem MPRF could be involved in CS induced by opioid withdrawal in an injury-free state. To date, there are no such "injury-free" human models of CS, rather behavioral hyperalgesia is induced by a peripheral conditioning stimulus such as topical or intradermal capsaicin, ultraviolet skin burns, and intradermal electrical stimulation (Klein et al., 2005). Furthermore, these are surrogate models representing only some features of neuropathic pain and may not represent pain in FPS where there is pain in the absence of demonstrable peripheral injury.

By using opioid withdrawal-induced hyperalgesia $(\mathrm{OIH})$ as an injury-free model in humans, we can gain insight into the mechanisms of FPS. Increasing such knowledge could lead to more effective treatment strategies for these patients. Furthermore, we do not know what constitutes the neural correlates underpinning $\mathrm{OIH}$ in humans, which is a clinical problem in its own right (Eisenach, 2000); especially where opioids are used liberally in the perioperative and acute pain management setting.

In the present study, we aim to induce hyperalgesia in a group of healthy humans during opioid withdrawal in the absence of a peripheral CS-inducing input, and to image the neural correlates 
using the noninvasive technique of functional magnetic resonance imaging (fMRI). This is a well established technique that has been used in studies that identified the MPRF as a neural correlate of capsaicin-induced CS in healthy humans (Lee et al., 2008). Therefore, we hypothesized that this region of the descending pain modulatory system of the brainstem would be involved.

\section{Materials and Methods}

\section{Subject recruitment and selection}

After obtaining Oxfordshire Research Ethics Committee (06/Q1605/37) approval, 33 healthy human subjects (American Society of Anesthesiologists physical status I) were recruited. Of these 33 subjects, 15 were females. After obtaining written informed consent, subjects attended a preliminary session where they received an infusion of remifentanil to the same maximum dose and duration to that which they would receive in the scanner. Only those subjects tolerant to the drug and the study procedures were invited to participate in the rest of the study.

\section{Study design}

After the preliminary visit we used a crossover design with two further visits, separated by at least one week. Subjects received an infusion of remifentanil during one of the visits and a saline infusion during the other. The subjects were blinded to the treatment. The visits were balanced for order.

\section{Experimental procedure}

All subjects fasted for $6 \mathrm{~h}$ on each study visit. On arrival, an intravenous cannula was placed in the subject's left forearm. Once the subject was laid supine in the MRI scanner, the infusion was connected to the cannula. Continuous monitoring of pulse rate (PR), blood oxygen saturation $(\mathrm{SpO} 2)$, respiratory rate $(\mathrm{RR})$, and end-tidal carbon dioxide partial pressures $\left(\mathrm{P}_{\mathrm{ET}} \mathrm{CO}_{2}\right)$ via nasal cannulae (Salter Labs) was commenced. Oxygen $1-21 / \mathrm{min}$ was delivered via the nasal cannulae throughout the experiment.

\section{Drug infusion}

A target-controlled infusion (TCI) pump delivered remifentanil intravenously. The TCI pump was preprogrammed with the threecompartment pharmacokinetic model of remifentanil that was published previously by Minto et al. (1997a,b). It controls the infusion rate of remifentanil to achieve and maintain a desired remifentanil effect site concentration, based on the subject's gender, age, weight, and height. A steady-state effect site concentration of $2 \mathrm{ng} \mathrm{ml}^{-1}$ for $30 \mathrm{~min}$ was used. Total duration of the infusion was $40 \mathrm{~min}$, which allowed $10 \mathrm{~min}$ to reach the steady effect site concentration.

\section{Noxious stimulation}

Thermal stimuli. An in-house developed thermal resistor with a fast rise time $\left(30^{\circ}\right.$ rise in $\left.0.8 \mathrm{~s}\right)$ was used to deliver noxious thermal stimuli via a thermode (Wise et al., 2002) attached to the medial-volar aspect of the proximal right forearm. The temperature of the thermode was adjusted adaptively to produce a pain intensity rating of 5 on a numerical rating scale (NRS) where 0 corresponds to "no pain" and 10 to "severe pain." The same temperature was used for all stimuli during that visit. These were delivered in blocks of 10 with an interstimulus interval of 55-68 s. Each stimulus lasted for $3 \mathrm{~s}$. The subjects recorded the pain intensity of each stimulus (as experienced at the time of the noxious stimulus) $\sim 15 \mathrm{~s}$ after the stimulus, using a visual analog scale (VAS) displayed on a computer screen where the anchors were "no pain" and "severe pain." There were four thermal stimulation blocks. These were delivered before the infusion (Th), during the infusion (Th2), $20 \mathrm{~min}$ after the infusion (Th3), and $45 \mathrm{~min}$ after the infusion (Th4). The experimental paradigm is outlined in Figure 1.
Punctate stimuli. Punctate stimuli were delivered to an area of skin $(6 \times 4 \mathrm{~cm})$ on the anteromedial aspect of the right leg $10 \mathrm{~cm}$ above the medial malleolus. We used non-skin penetrating punctate probes with a flat tip (Ziegler et al., 1999). This delivered a force of $512 \mathrm{mN}$. There were three punctate stimulation blocks. Each block consisted of a total of 10 stimuli, each with a duration of $\sim 2 \mathrm{~s}$. After each stimulus, subjects rated the perception (as perceived at the time the stimulus was delivered) using a VAS scale displayed on a computer screen. First they rated the intensity of the sensation felt ( $15 \mathrm{~s}$ after the stimulus) where the anchors were "not intense" and "extremely intense." Then they rated the intensity of the pain perceived ( $24 \mathrm{~s}$ after the stimulus) where the anchors were "no pain" and "severe pain."

To minimize sensitization of skin, application of successive stimuli were varied within the predetermined $6 \times 4 \mathrm{~cm}$ skin area. The same researcher delivered punctate stimuli manually.

Punctate stimulation blocks were delivered before the infusion (P1), during the infusion (P2), and $\sim 30 \mathrm{~min}$ after the infusion (P3). The experimental paradigm is given in Figure 1.

\section{Mood scale}

The 16-item Bond-Lader VAS (Bond et al., 1974) was used to track the mood changes during each visit. These items recorded the following aspects of the mood: tranquility, sociability, mental sedation, and physical sedation. Mood was recorded at baseline (M1) before commencing the infusion. Mood was also recorded during the infusion period on reaching steady state (M2a) and just before the end of the infusion (M2b). During the withdrawal period, mood was recorded $\sim 20 \mathrm{~min}$ (M3a) and $55 \mathrm{~min}$ (M3b) after stopping the infusion. The average of M2a and M2b were assumed to depict the mood during the infusion. The average of $\mathrm{M} 3 \mathrm{a}$ and $\mathrm{M} 3 \mathrm{~b}$ were assumed to depict the mood during the withdrawal period.

\section{fMRI data acquisition}

MRI data were acquired using a 3T Varian-Siemens whole-body magnetic resonance scanner. A head-only gradient coil was used with a birdcage radiofrequency coil for pulse transmission and signal reception. A whole-brain (including the midbrain, pons, rostral most medulla, and cerebellum) gradient-echo, echo-planar-imaging (EPI) sequence was used for functional scans (echo time, $30 \mathrm{~ms} ; 42$ contiguous $3.5 \mathrm{~mm}$-thick slices; field of view, $224 \times 224 \mathrm{~mm}$; matrix $64 \times 64$ ) with a repetition time of $3 \mathrm{~s}$ and 210 volumes in each thermal and 192 volumes in each punctate functional scan collection. The first four volumes were discarded to permit equilibration of the blood oxygen level-dependent (BOLD) signal. Fieldmaps were obtained using symmetric-asymmetric spin-echo sequence after the baseline functional scans ( $30 \mathrm{~ms}$ echo time, $0.5494 \mathrm{~ms}$ dwell time, field of view and matrix identical to EPI). A T1weighted structural $\left(1 \mathrm{~mm}^{3}\right.$ voxel $)$ image was acquired for the registration of statistical activation maps. 


\section{Data analysis}

Detection of subjects who develop hyperalgesia. We used within-subject data to identify the subjects who developed significant hyperalgesia in the postinfusion period. Emerging evidence suggest that not all subjects develop hyperalgesia during opioid withdrawal (Jensen et al., 2009). Therefore, in our study, evidence of OIH to thermal and punctate stimuli was assumed if there was a significant increase in intensity scores in at least one of the postinfusion stimulation blocks of the remifentanil visit when compared with the corresponding stimulation blocks in the saline visit ( $p<0.05$; two-tailed paired $t$ test). Only subjects who had a positive and statistically significant score based on the formula $\left(X_{\text {postinfusion (opioid) }}-\right.$ $\left.X_{\text {preinfusion (opioid) }}\right)>\left(X_{\text {postinfusion (saline) }}-X_{\text {preinfusion (saline) }}\right)$, where $(X)$ is the pain score, were considered to have developed hyperalgesia.

Based on this method we divided the whole group into two, those who developed hyperalgesia (responders) and those who did not (nonresponders).

Detection of differences in baseline data. After identifying the responders and nonresponders, we tested for differences in the following baseline data between the two visits within the subgroups as well as between the subgroups within each visit: temperatures used, noxious stimulus intensity, the four aspects of the mood scale, and the four cardio respiratory variables.

D'Agostino and Pearson omnibus normality test was used to examine the distribution of the data. For normally distributed data, paired twotailed $t$ test was performed for within-group comparisons. Two-tailed unpaired $t$ test was used for between-group comparisons. One-sample two-tailed $t$ tests were performed to evaluate whether the distribution of the opioid infusion and withdrawal effects were significantly different from a mean of zero. For data that were non-normally distributed we used the Wilcoxon signed rank test. The Mann-Witney test was used for between-group comparisons.

Detection of opioid-induced changes in psychophysical data. The effect of opioid infusion for a given set of psychophysical data $(X)$ was defined by $\left(X_{\text {infusion (opioid) }}-X_{\text {preinfusion (opioid) }}\right)-\left(X_{\text {infusion (saline) }}-X_{\text {preinfusion (saline) }}\right)$.

The effect of opioid withdrawal for a given set of psychophysical data $(X)$ was defined by $\left(X_{\text {postinfusion (opioid) }}-X_{\text {preinfusion(opioid) }}\right)-$ $\left(X_{\text {postinfusion (saline) }}-X_{\text {preinfusion (saline) }}\right)$. For the thermal data, the average of the two postinfusion period time points was taken.

Subtracting the preinfusion values from the postinfusion values accounts for the baseline differences between the visits. Subtracting the differences during the saline visit from those during the opioid visit accounts for the effect of time.

Correlation analysis. Where there are significant opioid withdrawalinduced effects on noxious stimulus perception and mood, a bivariate correlation was used to evaluate the relationship between these effects. These correlations were performed separately for responders and nonresponders.

fMRI data. Imaging data were analyzed using FEAT (FMRI Expert Analysis Tool) Version 5.98, part of FSL (Oxford Centre for Functional Magnetic Resonance Imaging of the Brain Software Library; www.fmrib. ox.ac.uk/fsl). The following preprocessing steps were applied: motion correction (Jenkinson et al., 2002), field-map correction of EPI distortion (Jenkinson, 2003; Jenkinson, 2004), removal of nonbrain voxels (Smith, 2002), spatial smoothing using a Gaussian kernel of full-width at half-maximum $5 \mathrm{~mm}$, grand-mean intensity normalization of the entire four-dimensional dataset by a single multiplicative factor, and highpass temporal filtering (Gaussian-weighted least-squares straight line fitting, with $\sigma=100.0 \mathrm{~s}$ ). Time-series statistical analysis was performed with local autocorrelation correction (Woolrich et al., 2001).

First level analysis. Statistical analysis was performed for each of the functional scans for individual subjects using a general linear modeling approach (Friston et al., 1995). The input stimulus functions related to noxious stimulation and the task of ratings were convolved with the gamma hemodynamic function (mean lag, $6 \mathrm{~s}$; full-width at half-height, $6 \mathrm{~s})$ to yield regressors for the general linear model that described the BOLD activity in the functional scans. The estimated motion parameters for each subject were included as covariates of no interest to reduce spurious activations due to head motion and scanner drift, thereby increasing statistical sensitivity. These analyses generated the parameter estimates (PE) for the regressors that described the BOLD activity evoked by noxious stimulation for each stimulation block. Registration of functional images to each subject's high resolution $\mathrm{T}_{1}$ scan, and then to the Montreal Neurological Institute (MNI) standard brain was performed using FLIRT (Linear Image Registration Tool, Centre for Functional Magnetic Resonance Imaging) (Jenkinson et al., 2002; Jenkinson and Smith, 2001).

Second level analysis. We first searched for differences in brain activity evoked by thermal stimulation in the baseline periods before administration of remifentanil and saline infusion. Statistical maps representing these differences were generated for the responder group and nonresponder group using paired tests that used FMRIB local analysis of mixed effects (FLAME I) (Woolrich et al., 2004). A cluster-based correction ( $Z$ score $>2.3, p<0.05$ ) was used for these maps to detect significant differences across the brain.

As for the psychophysical data, we used the same concept to determine the effects of opioid withdrawal on the neuronal activity (BOLD response). The effect of opioid withdrawal on neuronal activity $(X)$ in response to noxious stimulation was defined as $\left(X_{\text {postinfusion (opioid) }}-\right.$ $\left.X_{\text {preinfusion (opioid) }}\right)-\left(X_{\text {postinfusion (saline) }}-X_{\text {preinfusion (saline) }}\right)$. For neuronal response to thermal stimulation, the average statistical map of the two postinfusion period stimulation blocks (Th3 and Th4) was used. As the basis of central sensitization is neuronal plasticity rather than transient neuronal changes, we combined Th3 and Th4 when analyzing the imaging data (Woolf and Salter, 2000). For individual subjects, we generated the appropriate statistical contrast maps (fixed effects) using the outputs from the first level analyses. The map generated at second level represented the effect of opioid withdrawal on BOLD response (as defined above) across all voxels in the brain for that individual subject.

Third level analysis. We performed FLAME I (Woolrich et al., 2004) to create the group mean maps of the effect of opioid withdrawal for the responders and nonresponders. The individual subjects statistical maps generated at second level were used for this analysis.

To test our hypothesis, which is based on existing evidence for the role of the brainstem in OIH (Vanderah et al., 2001) in animals and in states of CS in humans (Lee et al., 2008; Zambreanu et al., 2005), we performed a region of interest analysis (ROI) of the brainstem. We used the functionally defined mask of the MPRF that showed increased activity in CS in the study by Lee et al. (2008). We performed a small volume correction (threshold free cluster enhanced at $p<0.05)$ (Smith and Nichols, 2009) using nonparametric permutation testing (5000 permutations) (Nichols and Holmes, 2002) for this ROI analysis.

ROI analyses. To further examine the behavior of the voxels that showed an effect of opioid withdrawal in the responders, we extracted the BOLD signal responses (percentage signal changes) from the voxels in this region. We limited this search to an area which contained voxels that showed increased activity within a sphere of $5 \mathrm{~mm}$ radius around the voxel with peak activity. For each subject, the BOLD responses to noxious stimulation in these isolated voxels during remifentanil and saline infusions were calculated as a mean percentage signal change. As with the psychophysical data, we performed one-sample two-tailed $t$ tests to evaluate whether the distribution of the opioid withdrawal-induced effects on the isolated voxels differed significantly from a normal distribution with a mean of zero. These were performed for both responders and nonresponders. Two-tailed unpaired $t$ tests were performed to evaluate differences in the opioid withdrawal-induced effects on the isolated voxels between responders and nonresponders.

Correlation analyses. Pearson $r$ was used to evaluate the correlations between the effects of opioid withdrawal on the activity of the isolated voxels and on pain and mood. The correlations were performed separately for responders and nonresponders.

\section{Results}

\section{Psychophysical data}

Detection of hyperalgesia in the opioid withdrawal period

Thirty-three subjects were recruited and 25 subjects were scanned. Of the eight subjects not scanned, five were excluded due to opioid-induced nausea and vomiting during the preliminary session, one subject was intolerant to the intravenous can- 
Table 1. Detection of subjects with hyperalgesia

\begin{tabular}{ccccccccc}
\hline SUB & SpIN 3-1 & RpIN 3-1 & SpP 3-1 & RpP 3-1 & STh 3-1 & RTh 3-1 & STh 4-1 & RTh 4-1 \\
\hline 1 & -1.12 & $0.5^{*}$ & -1.08 & $1.26^{*}$ & 0.02 & -1.28 & -0.28 & -1.96 \\
2 & 0.0 & 0.34 & 0.02 & 1.04 & -0.40 & 0.20 & -0.38 & $-0.02^{* a}$ \\
3 & -0.44 & -0.5 & -0.2 & -0.42 & 0.32 & -0.42 & -0.62 & -1.62 \\
4 & 1.02 & -0.08 & 1.66 & 0.76 & -0.62 & $0.54^{* a}$ & -0.48 & 0.14 \\
5 & -0.38 & -0.86 & 0.16 & 0.14 & -0.32 & -0.62 & -0.48 & -0.28 \\
6 & -0.18 & -0.12 & 0.06 & -0.9 & 0.76 & 0.52 & 0.16 & 0.20 \\
7 & 0.56 & 0.24 & 1.06 & 0.26 & 0.02 & -0.78 & -0.66 & -0.82 \\
8 & -0.52 & 0.74 & -0.3 & 0.72 & -0.28 & $0.26^{* a}$ & -0.80 & $0.04^{* a}$ \\
9 & -0.74 & -0.28 & -0.44 & 0.34 & -0.08 & $0.42^{* a}$ & 0.42 & 0.08 \\
10 & -0.14 & $1.32^{*}$ & -0.16 & $1.3^{*}$ & 0.92 & $1.74^{* a}$ & 0.64 & $1.24^{* a}$ \\
11 & -0.12 & -0.78 & -0.44 & -0.42 & 0.26 & -0.28 & -0.22 & -0.78 \\
12 & 2.06 & 3.06 & -0.1 & $3.26^{*}$ & 0.02 & 0.72 & 0.22 & -0.60 \\
14 & -0.34 & 0.02 & -0.68 & -0.1 & -1.18 & $1.0^{* a}$ & -1.30 & $1.28^{* a}$ \\
15 & 1.26 & 0 & -0.12 & 0.38 & -0.97 & $1.6^{* a}$ & -0.09 & -0.20 \\
16 & -0.5 & -0.8 & -1.06 & -0.8 & 0.36 & 0.60 & 0.12 & 0.42 \\
17 & 2.02 & 0.94 & 2.22 & 0.96 & -0.28 & $0.86^{* a}$ & -0.14 & $0.32^{* a}$ \\
18 & -1.56 & -0.34 & 0.4 & -0.32 & 0.08 & 0.24 & 0.27 & 0.90 \\
19 & -0.12 & -0.36 & 0.12 & -0.72 & 0.62 & -0.70 & $\$$ & $\$$ \\
20 & 0 & 0.1 & 0.04 & 0.04 & -0.88 & $0.18^{* a}$ & -1.38 & $0.08^{* a}$ \\
21 & -0.54 & -0.44 & -0.26 & -0.26 & -0.12 & $1.22^{* a}$ & -1.48 & $0.54^{* a}$ \\
22 & -0.56 & -0.16 & -0.74 & -0.5 & 0.54 & $1.76^{* a}$ & 0.18 & $1.48^{* a}$ \\
23 & 1.4 & 0.86 & 0.4 & 0.44 & -0.08 & $0.58^{* a}$ & 0.46 & 0.10 \\
25 & 0.02 & -0.58 & 0.12 & -0.86 & 0.12 & 0.14 & 0.10 & -0.28 \\
\hline
\end{tabular}

Individual data from the 23 subjects (SUB) are given in this table. These data consist of the mean intensity changes of the 10 trials from baseline to the postinfusion period. Each column header identifies the intensity change given in that column. Punctate sensation intensity change during saline visit is indicated as SpIN and that during the remifentanil visit as RplN. Punctate pain intensity change during saline visit is indicated as SpP and that during the remifentanil visit as RpP. Thermal pain intensity change during saline visit is indicated as STh and that during the remifentanil visit as RTh. The asterisk indicates a statistically significant increase during the remifentanil visit when compared to the saline visit $\left({ }^{*} p \leq 0.05\right.$ ). Twelve out of the 23 subjects demonstrated thermal hyperalgesia in either Th3 or Th4 or both. Only three subjects showed a significant increase in response to punctate stimuli.

${ }^{a}$ Thermal hyperalgesia.

${ }^{5}$ Missing data.

nula, one subject was claustrophobic on being placed in the scanner, and one subject failed to attend both scanner sessions.

Of the 25 subjects scanned (mean age, 30 years; age range, 21-46 years; 11 females), data from two subjects (S13 and S24) were excluded from analysis. S13 was excluded because the average threshold temperature of $41^{\circ} \mathrm{C}$ was well below the nociceptive threshold and more than two SDs lower than the rest of the subjects. S24 was excluded because of delays in the postinfusionscanning period of the second visit due to equipment malfunction. This meant that the postinfusion-scanning blocks were not performed at the same time in the two visits.

From the remaining 23 subjects, 12 subjects demonstrated hyperalgesia to thermal stimuli (responders). Details are given in Table 1. Only three of these subjects showed hyperalgesia to punctate stimuli. Therefore punctate data were not analyzed further.

\section{Baseline psychophysical variables}

There were no significant subgroup or visit influences on the following baseline variables: temperature and noxious stimulus perception, and mood or cardio respiratory variables. Group mean values and the \pm SD of these variables in responders and nonresponders are tabulated in Table 2 for the baseline saline and remifentanil visits.

\section{Opioid-induced changes in psychophysical data}

Changes in perception of thermal noxious stimuli. During the infusion period, in responders and nonresponders there is a significant opioid-induced reduction of thermal pain intensity $(p<$ 0.001 ) in responders and nonresponders (Fig. 2). There is no
Table 2. Baseline psychophysical variables

\begin{tabular}{|c|c|c|c|c|}
\hline \multirow[b]{2}{*}{ Psychophysical variables } & \multicolumn{2}{|l|}{ Responders } & \multicolumn{2}{|c|}{ Nonresponders } \\
\hline & Saline & Remifentanil & Saline & Remifentanil \\
\hline Temperature $\left(\mathrm{C}^{\circ}\right)$ & $50.5( \pm 1.5)$ & $50.2( \pm 1.8)$ & $51.1( \pm 1.9)$ & $51.1( \pm 2.2)$ \\
\hline Thermal pain (VAS) & $5.3( \pm 0.1)$ & $5.1( \pm 1.2)$ & $4.9( \pm 0.4)$ & $5.1( \pm 0.6)$ \\
\hline Punctate pain (VAS) & $2.3( \pm 1.4)$ & $2.4( \pm 1.4)$ & $2.1( \pm 1.2)$ & $2.1( \pm 1.5)$ \\
\hline Punctate intensity (VAS) & $3.7( \pm 1.2)$ & $4.0( \pm 1.0)$ & $3.7( \pm 1.4)$ & $3.4( \pm 0.9)$ \\
\hline Tranquility (VAS) & $7.3( \pm 1.6)$ & $7.4( \pm 1.5)$ & $7.8( \pm 1.5)$ & $7.6( \pm 1.8)$ \\
\hline Sociability (VAS) & $6.7( \pm 1.3)$ & $6.7( \pm 1.5)$ & $7.3( \pm 1.4)$ & $7.6( \pm 1.4)$ \\
\hline Physical sedation (VAS) & $3.0( \pm 1.1)$ & $3.1( \pm 1.5)$ & $2.9( \pm 1.5)$ & $2.5( \pm 1.8)$ \\
\hline Mental sedation (VAS) & $2.9( \pm 2.0)$ & $3.2( \pm 1.8)$ & $3.4( \pm 1.7)$ & $2.5( \pm 2.4)$ \\
\hline End tidal $\mathrm{CO}_{2}(\mathrm{mmHg})$ & $41.3( \pm 3.1)$ & $40.4( \pm 3.9)$ & $40.6( \pm 5.2)$ & $40.8( \pm 3.7)$ \\
\hline Respiratory rate (breaths/min) & $15( \pm 3)$ & $16( \pm 3)$ & $15( \pm 3)$ & $14( \pm 3)$ \\
\hline Hemoglobin saturation (\%) & $98.4( \pm 0.8)$ & $98.4( \pm 1.4)$ & $98.3( \pm 0.7)$ & $98.8( \pm 0.2)$ \\
\hline Pulse rate (beats/min) & $59( \pm 8)$ & $58( \pm 8)$ & $55( \pm 6)$ & $58( \pm 6)$ \\
\hline
\end{tabular}

The baseline psychophysical variables measured were the temperature of the stimulus, intensity of the noxious stimuli (thermal pain, punctate pain, and punctate stimulus), mood (tranquility, sociability, physical sedation, and mental sedation), and cardio-respiratory measures (end-tidal $\mathrm{CO}_{2}$, respiratory rate, hemoglobin saturation, and pulse rate). Group mean values and the $\pm S D$ of these variables in responders and nonresponders at baseline in the saline visit and remifentanil visit are shown. Measurement units are shown within the parentheses in column 1. There are no significant subgroup or visit influences on any of these variables.

significant difference in the magnitude of this opioid infusioninduced effect between responders and nonresponders.

During the postinfusion period, statistical tests regarding effects of opioid withdrawal on thermal pain intensity were not performed, as these effects were predetermined in the selection of responders and nonresponders.

Changes in the mood. During the infusion period, both responders and nonresponders are significantly more sedated, both mentally and physically, due to the opioid (mental sedation in responders $p<0.05$ and in nonresponders $p<0.01$; physical sedation in responders and nonresponders $p<0.05$ ) (Fig. 2). However, the magnitudes of these opioid-induced changes are not significantly different between the groups. There is no significant opioid-induced effect on tranquility and sociability in both groups during the infusion.

During the postinfusion period, opioid withdrawal-induced loss of tranquility is significant only in responders $(p<0.01)$. Both groups are significantly less sociable due to the opioid withdrawal $(p<0.05)$. However, the magnitudes of these opioid withdrawal-induced changes are not statistically different between the groups. In both groups there are no significant opioid withdrawal-induced changes in mental and physical sedation.

Changes in cardio respiratory data. During the infusion period, in both responders and nonresponders, the opioid infusion induced significantly lower respiratory rates $(p<0.01$ in both groups). The mean opioid-induced reduction $( \pm S D)$ for responders is $4.5( \pm 3)$ breaths/min while that for nonresponders is $3( \pm 2)$ breaths $/ \mathrm{min}$. The opioid also induced higher end-tidal $\mathrm{CO}_{2}$ levels $\left(\mathrm{P}_{\mathrm{ET}} \mathrm{CO}_{2}\right)$ in responders and nonresponders $(p<0.01$ in both groups). The mean opioid-induced increase $( \pm S D)$ for responders is $5.4( \pm 3.52) \mathrm{mmHg}$ while that for nonresponders is $6.1( \pm 3.19) \mathrm{mmHg}$. Only nonresponders developed a statistically significant $(p<0.05)$ but clinically insignificant reduction in percentage oxygen saturation $\left(\mathrm{SpO}_{2}\right)$ due to the opioid. The mean opioid-induced reduction $( \pm \mathrm{SD})$ for responders is 0.2 $( \pm 1.44) \%$ while that for nonresponders is 0.8 ( \pm 0.9$) \%$. There are no significant opioid-induced changes in the pulse rate in both groups. The magnitudes of these opioid-induced changes in the cardiorespiratory indices are not significantly different between the groups.

During the postinfusion period, no significant opioid withdrawal-induced changes are present in the cardio respiratory variables in both groups. 

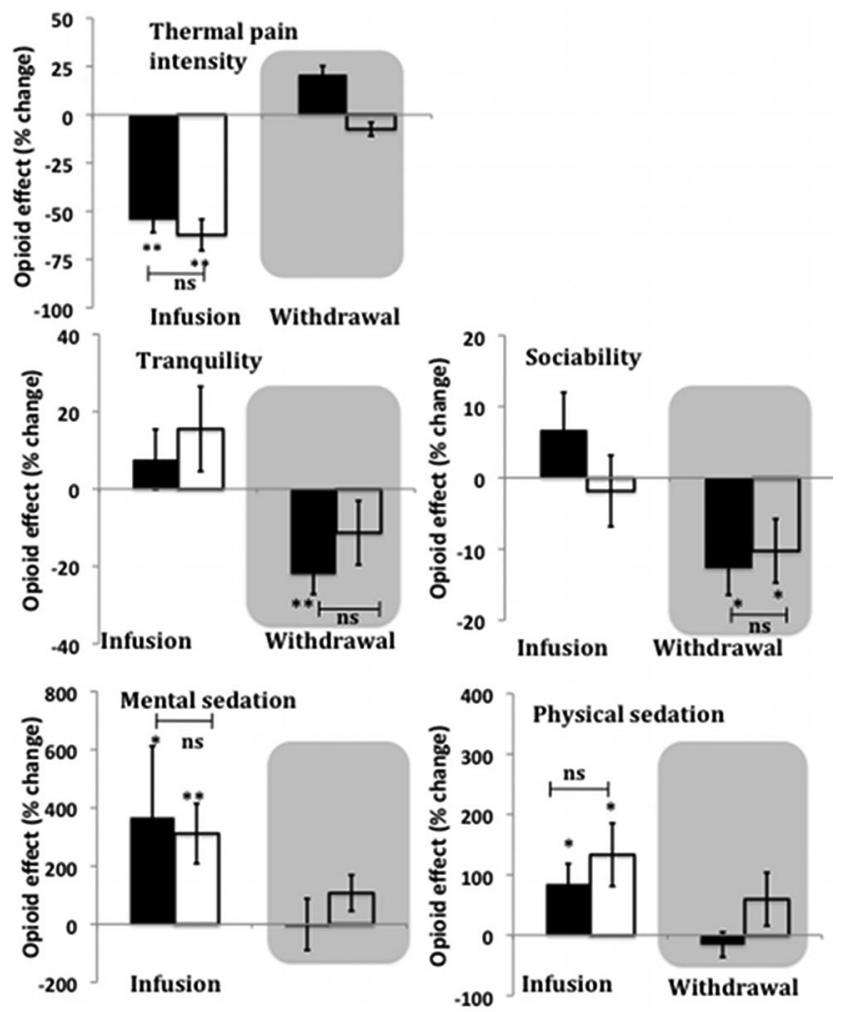

Figure 2. Effect of opioid infusion and withdrawal on perception of thermal noxious stimuli (top row) and mood (middle and bottom rows). The $y$-axis shows opioid effect on perceived thermal pain intensity and mood. The effect of opioid infusion for thermal pain intensity and $\operatorname{mood}(X)$ were defined by $\left(X_{\text {during-infusion (opioid) }}-X_{\text {preinfusion(opioid) }}\right)-\left(X_{\text {during infusion (saline) }}-\right.$ $X_{\text {preinfusion (saline) }}$ ). The effect of opioid withdrawal for a thermal pain intensity and $\operatorname{mood}(X)$ were defined by $\left(X_{\text {postinfusion (opioid) }}-X_{\text {preinfusion (opioid) }}\right)-\left(X_{\text {postinfusion (saline) }}-X_{\text {preinfusion (saline) }}\right)$. Response of responders (black bars) and nonresponders (open bars) are shown. Withdrawal period is shown in the gray shaded area. Error bars indicate SEM. A significant opioid effect is shown as * $p<$ $0.05,{ }^{* *} p<0.01$. Between-group comparisons are shown where there is a significant opioid effect. (ns, Nonsignificant). Significant opioid-induced analgesia was observed in both groups (top row). However, there is no significant difference between the groups in the magnitude of these effects. Statistical tests for the effects of opioid withdrawal on thermal pain were not performed, as these effects were predetermined in the selection of responders and nonresponders. Significant opioidinduced increases in mental and physical sedation were observed in both groups (bottom row). There is no significant difference in the magnitude of these effects between the two groups. There are no significant opioid-induced effects on tranquility and sociability in both groups (middle row). The loss of tranquility induced by opioid withdrawal is significant only in responders. Both groups are significantly less sociable due to opioid withdrawal. There is no significant difference in the magnitude of these effects between the two groups. There is no significant effect of opioid withdrawal on mental and physical sedation in both groups.

Correlation analysis of opioid withdrawal-induced thermal hyperalgesia and mood changes. In responders, significant opioid withdrawal-induced effects are observed in thermal pain perception, tranquility, and sociability. However, the opioid withdrawal-induced effect of thermal hyperalgesia does not correlate with the loss of tranquility and sociability during this period. No significant correlation is observed in nonresponders either.

\section{fMRI data}

Baseline neuronal responses

There are no significant differences in the neuronal response to noxious thermal stimulation between the baseline periods of the remifentanil and saline visits, either in responders or nonresponders across all voxels in the brain (cluster-based correction with $Z$ score $>2.3, p<0.05$ ). This concurs with baseline psycho- physical data, which show no differences between visits in either group.

\section{Opioid-induced effects on neuronal response (BOLD activity) in} $M P R F$ region

In responders only, there is a significant rise in activity from baseline in response to thermal stimulation during the opioid withdrawal period (after accounting for the effects of time) in a cluster of voxels in the MPRF (Fig. 3). This area of activity lies within the same area that we have previously demonstrated to be involved in the maintenance of central sensitization induced by capsaicin in humans (Lee et al., 2008). This opioid withdrawalinduced relative change in activity in isolated MPRF voxels in responders is significantly higher compared with nonresponders (Fig. 4). Furthermore, during opioid infusion in responders there is a trend toward an increased relative change of BOLD activity in isolated voxels of the MPRF, but in nonresponders a trend toward a decreased relative change is observed; these changes are not significantly different from each other.

Additionally, the responders show a significant rise in the neuronal response to thermal noxious stimulation in the MPRF from baseline to the postinfusion period during the remifentanil visit when compared with the nonresponders. However, there is no such difference in change in MPRF activity from the baseline to the postinfusion period during the saline visit in both responders and nonresponders (Fig. 5).

Correlation analysis of the opioid withdrawal-induced effects In responders, opioid withdrawal induced thermal hyperalgesia, a loss of tranquility and sociability and an increase in BOLD activity in an area of the MPRF. There is a significant negative correlation ( $r=0.61, p=0.03$ ) between the opioid withdrawalinduced increase in pain perception and increase in BOLD activity in the MPRF region (Fig. 6). There is no such correlation in the nonresponders.

In both groups, there are no significant correlations between the MPRF activity and the two mood variables (tranquility and sociability) that had significant opioid withdrawal-induced effects (data not shown as figures).

\section{Discussion}

In a cohort of healthy subjects, we have demonstrated thermal hyperalgesia without a peripheral injury model induced by acute opioid withdrawal. This was accompanied by a reduction in tranquility and sociability and a relative increased activity in the MPRF. This is the same area in which Lee et al. (2008) observed increased activity in CS induced by capsaicin in healthy volunteers that was specific to the state of CS, as a perception-matched stimulus in the nonsensitized state failed to produce the same increased MPRF activity. We did not observe significant opioidinduced mechanical hyperalgesia, suggesting differences between models of CS induced by capsaicin and opioid withdrawal.

Our study provides the first clear evidence from humans that the brainstem MPRF plays a key role in the expression of hyperalgesia without peripheral injury induced by opioid withdrawal; i.e., an injury-free model of CS in humans.

A recent healthy volunteer study demonstrated also thermal hyperalgesia during opioid withdrawal in the absence of tissue injury (Jensen et al., 2009). In the absence of a control arm this hyperalgesia cannot be attributed conclusively to the effects of opioid withdrawal as it could also represent the effect of repeated thermal stimulation. Our study methodology includes a control arm. We have defined all "opioid-induced" effects after excluding effects of repeated thermal stimulation using this control arm. 


\section{Role of the MPRF in OIH}

In responders, OIH showed a significant negative correlation with the opioid withdrawal-induced relative increase in activity of the MPRF (Fig. 6). Neither of the opioid withdrawal-induced reductions in mood correlated with this relative increased MPRF activity. This suggests a lack of involvement of the MPRF in the opioid withdrawal-induced effects on mood.

The negative correlation of MPRF activity and pain perception in responders implies that, when increased during opioid withdrawal, the MPRF activity reflects a net descending inhibitory response to the hyperalgesia induced by opioid withdrawal. Such a relationship is absent in the nonresponders, indicating that this inhibitory effect is specific to the state of $\mathrm{OIH}$ that occurs in susceptible individuals.

MPRF contains nuclei, such as the nucleus cuneiformis. These nuclei are structurally and functionally similar to those in the rostral ventromedial medulla (RVM) with facilitatory "ON cells" and inhibitory "OFF cells" (Zemlan and Behbehani, 1988; Haws et al., 1989; Fields et al., 2006). Therefore, such isolated increased MPRF activity would represent the activity of both groups of cells. Evidence from rodent studies indicate that descending inhibitory and facilitatory influences can be simultaneously engaged from nuclei in the RVM, with these influences being conveyed in different spinal funiculi (Zhuo and Gebhart, 1997; Pinto-Ribeiro et al., 2008). Therefore, opioid withdrawalinduced increased MPRF activity in our study could represent increased activity in both groups of cells with the OFF cell activity predominating. Behaviorally, this OFF cell activity manifests as an inhibitory influence on the thermal hyperalgesia. However, we cannot ascertain the amount of activity of these cells without selectively inactivating specific ON and OFF cells in the MPRF. Ours is an fMRI study that measures the neuronal activity induced by opioid withdrawal in response to brief noxious stimuli. Therefore, based on our results we conclude that the brainstem nuclei within the MPRF are involved in regulating the expression $\mathrm{OIH}$ in the absence of tissue-injury.

Although it is possible that the MPRF is involved in the generation of the $\mathrm{OIH}$, it is more likely that it is responding to the $\mathrm{OIH}$ generated by other sites in the CNS, including other supra spinal and spinal sites (Ossipov et al., 2005; Vera-Portocarrero et al., 2007; Drdla et al., 2009).

There are opioid receptors in the peripheral nerves making it theoretically possible for the $\mathrm{OIH}$ to be induced peripherally. Although there is convincing evidence for peripherally induced opioid analgesia in humans (Kalso et al., 2002), convincing evidence for peripherally induced opioid hyperalgesia is lacking. Indeed, absence of analgesic tolerance/hyperalgesia is considered one of the main advantages of peripheral opioid agonists (Stein et al., 2009). Hyperalgesia induced via peripheral opioid receptors has mostly been demonstrated in the presence of inflammation/ injury (Aley et al., 1995), yet others contradict these findings (Tokuyama et al., 1998). Unlike the central opioid antagonists, the peripheral opioid antagonists when administered to humans a Opioid infusion induced effects

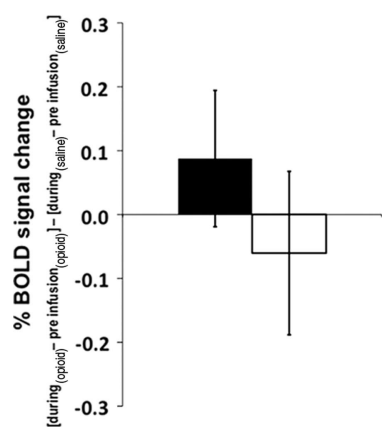

b Opioid withdrawal induced effects

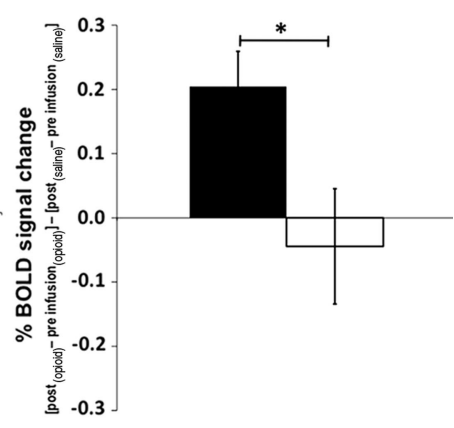

Figure 4. $\quad \boldsymbol{a}, \boldsymbol{b}$, 0pioid infusion-induced effects ( $\boldsymbol{a}$ ) and opioid withdrawal-induced effects (b) on neuronal response to thermal noxious stimulation within the isolated MPRF in responders (black bars) and nonresponders (open bars). The $y$-axes show the induced effects on neuronal response as percentage BOLD signal change. Opioid infusion-induced effect on neuronal response $(X)$ was defined by $\left(X_{\text {during-infusion (opioid) }}-X_{\text {preinfusion (opioid) }}\right)-\left(X_{\text {during-infusion (saline) }}-X\right.$ preinfusion (saline) $)$. Opioid withdrawal-induced effect on neuronal response $(X)$ was defined by $\left(X_{\text {postinfusion (opioid) }}-X_{\text {preinfusion(opioid) }}\right)-\left(X_{\text {postinfusion (saline) }}-X_{\text {preinfusion(saline) }}\right)$. Error bars indicate SEM. $\left({ }^{*} p<0.05\right)$. The opioid withdrawal-induced neuronal response to thermal noxious stimulation in the MPRF is significantly higher in responders than in nonresponders. The effect of the opioid infusion on BOLD activity in the MPRF appears to increase in responders and decrease in nonresponders, but these effects are not statistically significant.

receiving both acute and chronic opioid therapy failed to precipitate hyperalgesia (Wolff et al., 2004; Yuan and Israel, 2006), suggesting that $\mathrm{OIH}$ in humans is centrally induced. Therefore, based on a wealth of evidence from preclinical studies, the hyperalgesia to the systemic opioid administration in our study is most likely due to central sensitization. Although we cannot conclusively identify the location of the causal generator of hyperalgesia, it is clear from the data presented that the MPRF regulates the 


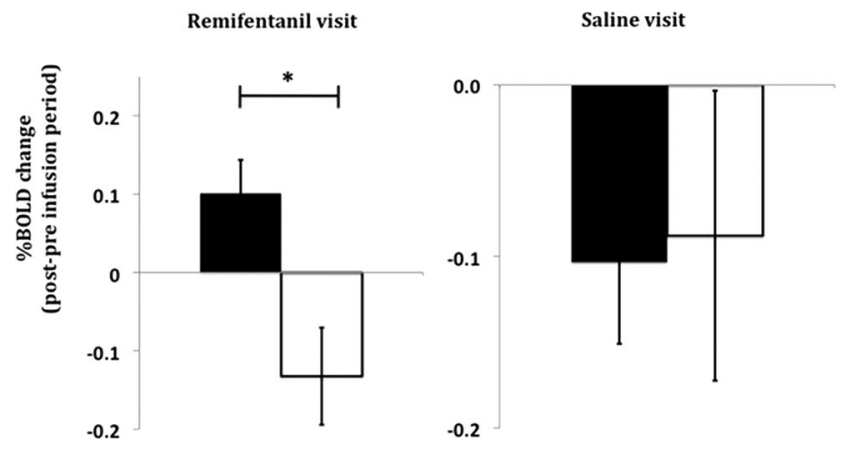

Figure 5. The change in neuronal response of the MPRF to thermal noxious stimulation in responders (black bars) and nonresponders (open bars). The $y$-axes show the average change in neuronal response from the preinfusion to postinfusion period during the remifentanil (left) and the saline visits as percentage BOLD signal change $\left({ }^{*} p<0.01\right)$.

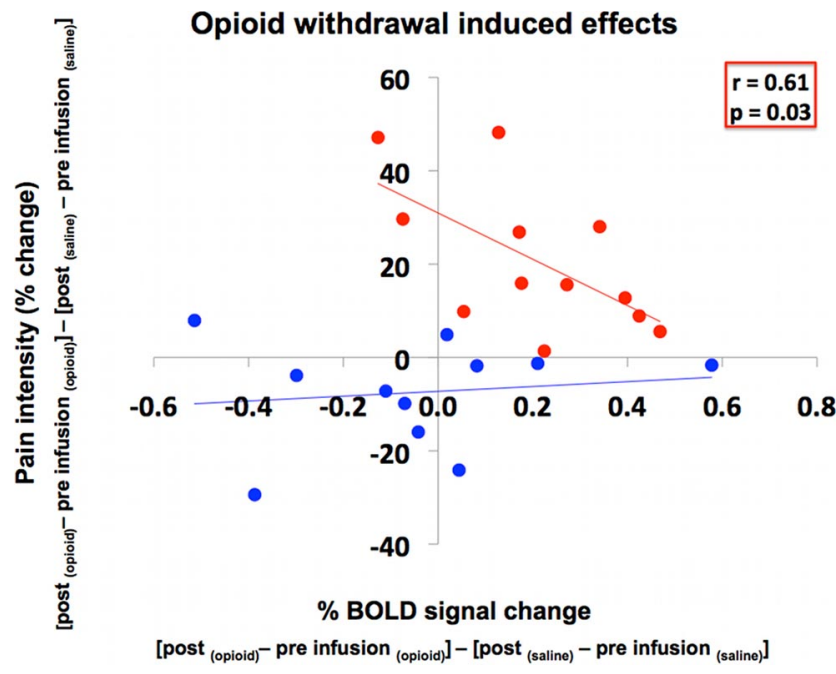

Figure 6. Correlation of opioid withdrawal-induced effects. This graph shows the correlation of the opioid withdrawal-induced effects on MPRF activity and pain in responders (red) and nonresponders (blue). A significant negative correlation $(r=0.61 ; p=0.03)$ is observed only in responders. The opioid withdrawal-induced effects on MPRF neuronal response (percentage BOLD signal change) and pain intensity to thermal noxious stimulation $(X)$ were defined by $\left(X_{\text {postinfusion (opioid) }}-X_{\text {preinfusion(opioid) }}\right)-\left(X_{\text {postinfusion (saline) }}-X_{\text {preinfusion(saline) }}\right)$.

expression of hyperalgesia in an opioid withdrawal-induced state of CS in the absence of tissue injury.

\section{Individual susceptibility to hyperalgesia during opioid} withdrawal in the absence of injury

It is intriguing why only a subset of participants developed opioid withdrawal-induced hyperalgesia accompanied by increased activity in the MPRF, while others did not. It is possible that given a higher dose and for a longer duration all the subjects could have developed the phenomenon. Dose dependency of this phenomenon has been suggested by some authors (Angst and Clark, 2006).

We used subjective pain scores of individual subjects to identify those subjects developing biologically significant OIH. Since pain scores were obtained after every stimulus (10 stimuli per block), we were able to perform statistical significance testing on the average score for each individual. Only subjects who have a positive and statistically significant score based on the formula $\left(X_{\text {postinfusion (opioid) }}-X_{\text {preinfusion (opioid) }}\right)>\left(X_{\text {postinfusion (saline) }}-\right.$ $X_{\text {preinfusion (saline) }}$ ), where $X$ is the pain score, were considered to have developed hyperalgesia. This firmly removes the possibility of a false positive result from sensitization due to repeated thermal stimuli and accounts for baseline effects. In the absence of an agreement about what might be considered as biologically significant OIH based on subjective pain scores, we believe ours is a logical and conservative method avoiding the use of an arbitrary cutoff score.

The presence of such individual susceptibility also highlights the need for larger study cohorts to detect $\mathrm{OIH}$ in an injury-free model. This could well be one of the reasons why two other studies with a cohort of 10 subjects (Angst et al., 2003; Hood et al., 2003) failed to demonstrate OIH in the uninjured skin.

In our study, opioid withdrawal induced a relative increased activity in the MPRF only in the responders (Figs. 3, 4). Interestingly, the mean MPRF activations are similarly reduced in both responders and nonresponders during the saline visit. The reduction remains in the nonresponders during opioid withdrawal, but the responders demonstrated an opposite increase (Fig. 5). These data strongly suggest that the groups form distinct populations in terms of the role of the MPRF during opioid withdrawal.

Another clue to the presence of a true biological difference between the groups comes from the different activity pattern of the MPRF induced by the opioid infusion itself (Fig. 4). Although statistically not significant, opioid infusion appears to induce a reduction in MPRF activity in the nonresponders but an increase in responders. This supports the notion that the response of the endogenous descending pain modulatory systems when exposed to an opioid is different (but not significantly so) in the two groups while experiencing similar degrees of behavioral analgesia (Fig. 2).

Emerging evidence from preclinical studies suggest a role for genetics in opioid-induced hyperalgesia (Nackley et al., 2006). Interestingly, hyperalgesia in the study by Jensen et al. (2009), which could be partly due to opioid withdrawal, was influenced by the common functional polymorphism (val ${ }^{158} \mathrm{met}$ ) of the COMT (catechol-O-methyltransferase) gene. Those subjects with the met/met polymorphism reported the highest pain intensity ratings, while subjects with the val/val polymorphism reported the lowest pain intensity ratings during the opioid withdrawal period. These observations support our finding of the presence of $\mathrm{OIH}$ only in a subset of participants where we used subjective pain scores of individual subjects to identify those developing biologically significant OIH.

\section{Conclusion}

In a cohort of susceptible healthy volunteers we have demonstrated OIH in an injury-free model of CS accompanied by a relative increased MPRF activity; an area previously shown to be a marker of CS states in healthy humans. The negative correlation between opioid withdrawal-induced MPRF activity and hyperalgesia suggest that the MPRF exerts an overall inhibitory effect regulating the expression of $\mathrm{OIH}$; so, a lack of inhibition from the MPRF during withdrawal may exacerbate the hyperalgesia in the susceptible. This is in keeping with the observation of abnormal descending inhibition in patients with FPS. Our study also highlights that there are differences in the susceptibility to the development of $\mathrm{OIH}$ along with differences in behavior of the MPRF. Further investigation of this phenomenon would help us to detect those at risk of developing $\mathrm{OIH}$ and chronic pain states, which is key to implementing suitable preventative strategies. 


\section{References}

Aley KO, Green PG, Levine JD (1995) Opioid and adenosine peripheral antinociception are subject to tolerance and withdrawal. J Neurosci 15:8031-8038.

Angst MS, Clark JD (2006) Opioid-induced hyperalgesia: a qualitative systematic review. Anesthesiology 104:570-587.

Angst MS, Koppert W, Pahl I, Clark DJ, Schmelz M (2003) Short-term infusion of the mu-opioid agonist remifentanil in humans causes hyperalgesia during withdrawal. Pain 106:49-57.

Berman SM, Naliboff BD, Suyenobu B, Labus JS, Stains J, Ohning G, Kilpatrick L, Bueller JA, Ruby K, Jarcho J, Mayer EA (2008) Reduced brainstem inhibition during anticipated pelvic visceral pain correlates with enhanced brain response to the visceral stimulus in women with irritable bowel syndrome. J Neurosci 28:349-359.

Bond AJ, James DC, Lader MH (1974) Physiological and psychological measures in anxious patients. Psychol Med 4:364-373.

Drdla R, Gassner M, Gingl E, Sandkühler J (2009) Induction of synaptic long-term potentiation after opioid withdrawal. Science 325:207-210.

Duvernoy H (1995) The human brain stem and cerebellum. In: Surface, structure, vascularization and three-dimensional anatomy with MRI. Vienna, Austria: Springer.

Eisenach JC (2000) Preemptive hyperalgesia, not analgesia? Anesthesiology 92:308-309.

Fields H, Basbaum A, Heinricher M (2006) Central nervous system mechanisms of pain modulation. In: Wall and Melzack's text book of pain (McMahon SB, Koltzenburg M, eds.) New York, NY: Elsevier, Churchill Livingstone.

Friston KJ, Frith CD, Frackowiak RS, Turner R (1995) Characterizing dynamic brain responses with fMRI: a multivariate approach. Neuroimage 2:166-172.

Gebhart GF (2004) Descending modulation of pain. Neurosci Biobehav Rev 27:729-737.

Haws CM, Williamson AM, Fields HL (1989) Putative nociceptive modulatory neurons in the dorsolateral pontomesencephalic reticular formation. Brain Res 483:272-282.

Heinricher MM, Tavares I, Leith JL, Lumb BM (2009) Descending control of nociception: specificity, recruitment and plasticity. Brain Res Rev 60:214-225.

Hood DD, Curry R, Eisenach JC (2003) Intravenous remifentanil produces withdrawal hyperalgesia in volunteers with capsaicin-induced hyperalgesia. Anesth Analg 97:810-815.

Iannetti GD, Zambreanu L, Wise RG, Buchanan TJ, Huggins JP, Smart TS, Vennart W, Tracey I (2005) Pharmacological modulation of painrelated brain activity during normal and central sensitization states in humans. Proc Natl Acad Sci U S A 102:18195-18200.

Jenkinson M (2003) Fast, automated, N-dimensional phase-unwrapping algorithm. Magn Reson Med 49:193-197.

Jenkinson M (2004) Improving the registration of B0-disorted EPI images using calculated cost function weights. Paper presented at Tenth International Conference on Functional Mapping of the Human Brain, Budapest, Hungary, June.

Jenkinson M, Smith S (2001) A global optimisation method for robust affine registration of brain images. Med Image Anal 5:143-156.

Jenkinson M, Bannister P, Brady M, Smith S (2002) Improved optimisation for the robust and accurate linear registration and motion correction of brain images. Neuroimage 17:825-841.

Jensen KB, Lonsdorf TB, Schalling M, Kosek E, Ingvar M (2009) Increased sensitivity to thermal pain following a single opiate dose is influenced by the COMT val(158)met polymorphism. PLoS One 4:e6016.

JiRR, Kohno T, Moore KA, WoolfCJ (2003) Central sensitization andLTP: do pain and memory share similar mechanisms? Trends Neurosci 26:696-705.

Julien N, Goffaux P, Arsenault P, Marchand S (2005) Widespread pain in fibromyalgia is related to a deficit of endogenous pain inhibition. Pain 114:295-302.

Kalso E, Smith L, McQuay HJ, Andrew Moore R (2002) No pain, no gain: clinical excellence and scientific rigour-lessons learned from IA morphine. Pain 98:269-275.

Kaplan H, Fields HL (1991) Hyperalgesia during acute opioid abstinence: evidence for a nociceptive facilitating function of the rostral ventromedial medulla. J Neurosci 11:1433-1439.

Klein T, Magerl W, Rolke R, Treede RD (2005) Human surrogate models of neuropathic pain. Pain 115:227-233.

Lee MC, Zambreanu L, Menon DK, Tracey I (2008) Identifying brain activ- ity specifically related to the maintenance and perceptual consequence of central sensitization in humans. J Neurosci 28:11642-11649.

Minto CF, Schnider TW, Egan TD, Youngs E, Lemmens HJ, Gambus PL, Billard V, Hoke JF, Moore KH, Hermann DJ, Muir KT, Mandema JW, Shafer SL (1997a) Influence of age and gender on the pharmacokinetics and pharmacodynamics of remifentanil. I. Model development. Anesthesiology 86:10-23.

Minto CF, Schnider TW, Shafer SL (1997b) Pharmacokinetics and pharmacodynamics of remifentanil. II. Model application. Anesthesiology 86:24-33.

Nackley A, Diatchenko L, Maixner W (2006) Perspectives on the genetic basis of opioid-induced hyperalgesia. Anesthesiology 104:909-910.

Nichols TE, Holmes AP (2002) Nonparametric permutation tests for functional neuroimaging: a primer with examples. Hum Brain Mapp 15:1-25.

Ossipov MH, Lai J, King T, Vanderah TW, Porreca F (2005) Underlying mechanisms of pronociceptive consequences of prolonged morphine exposure. Biopolymers 80:319-324.

Pinto-Ribeiro F, Ansah OB, Almeida A, Pertovaara A (2008) Influence of arthritis on descending modulation of nociception from the paraventricular nucleus of the hypothalamus. Brain Res 1197:63-75.

Smith SM (2002) Fast robust automated brain extraction. Hum Brain Mapp 17:143-155.

Smith SM, Nichols TS (2009) Threshold-free cluster enhancement: addressing problems of smoothing, threshold dependence and localisation in cluster inference. Neuroimage 44:83-98.

Stein C, Clark JD, Oh U, Vasko MR, Wilcox GL, Overland AC, Vanderah TW, Spencer RH (2009) Peripheral mechanisms of pain and analgesia. Brain Res Rev 60:90-113.

Tillisch K, Mayer EA (2005) Pain perception in irritable bowel syndrome. CNS Spectr 10:877-882.

Tokuyama S, Inoue M, Fuchigami T, Ueda H (1998) Lack of tolerance in peripheral opioid analgesia in mice. Life Sci 62:1677-1681.

Vanderah TW, Suenaga NM, Ossipov MH, Malan TP Jr, Lai J, Porreca F (2001) Tonic descending facilitation from the rostral ventromedial medulla mediates opioid-induced abnormal pain and antinociceptive tolerance. J Neurosci 21:279-286.

Vera-Portocarrero LP, Zhang ET, King T, Ossipov MH, Vanderah TW, Lai J, Porreca F (2007) Spinal NK-1 receptor expressing neurons mediate opioid-induced hyperalgesia and antinociceptive tolerance via activation of descending pathways. Pain 129:35-45.

Wise RG, Rogers R, Painter D, Bantick S, Ploghaus A, Williams P, Rapeport G, Tracey I (2002) Combining fMRI with a pharmacokinetic model to determine which brain areas activated by painful stimulation are specifically modulated by remifentanil. Neuroimage 16:999-1014.

Wolff BG, Michelassi F, Gerkin TM, Techner L, Gabriel K, Du W, Wallin BA. Alvimopan, a novel, peripherally acting mu opioid antagonist: results of a multicenter, randomized, double-blind, placebo-controlled, phase III trial of major abdominal surgery and postoperative ileus. Ann Surg 240: 728-734, 2004; discussion 734-725.

Woolf CJ, Salter MW (2000) Neuronal plasticity: increasing the gain in pain. Science 288:1765-1769.

Woolrich MW, Ripley BD, Brady M, Smith SM (2001) Temporal autocorrelation in univariate linear modeling of FMRI data. Neuroimage 14:1370-1386.

Woolrich MW, Behrens TE, Beckmann CF, Jenkinson M, Smith SM (2004) Multilevel linear modelling for FMRI group analysis using Bayesian inference. Neuroimage 21:1732-1747.

Yuan CS, Israel RJ (2006) Methylnaltrexone, a novel peripheral opioid receptor antagonist for the treatment of opioid side effects. Expert Opin Investig Drugs 15:541-552.

Yunus MB (2007) Fibromyalgia and overlapping disorders: the unifying concept of central sensitivity syndromes. Semin Arthritis Rheum 36:339-356.

Zambreanu L, Wise RG, Brooks JC, Iannetti GD, Tracey I (2005) A role for the brainstem in central sensitisation in humans. Evidence from functional magnetic resonance imaging. Pain 114:397-407.

Zemlan FP, Behbehani MM (1988) Nucleus cuneiformis and pain modulation: anatomy and behavioral pharmacology. Brain Res 453:89-102.

Zhuo M, Gebhart GF (1997) Biphasic modulation of spinal nociceptive transmission from the medullary raphe nuclei in the rat. J Neurophysiol 78:746-758.

Ziegler EA, Magerl W, Meyer RA, Treede RD (1999) Secondary hyperalgesia to punctate mechanical stimuli. Central sensitization to A-fibre nociceptor input. Brain 122: 2245-2257. 